\title{
THE EXTERNAL INTELLIGENT INFRASTRUCTURE
}

\author{
Dag Karlsen and Frank Lillehagen \\ Computas AS, NORWAY, \\ dk@computas.com, fli@computas.com
}

Dag Runar Elvekrok Det Norske Veritas AS, NORWAY, dag.runar.elvekrok@dnv.com

\begin{abstract}
Intelligent Infrastructures play an increasingly important role in the formation and operation of Extended Enterprises (EE), Virtual Organizations (VO) and Collaborative Businesses (CB). The main purpose of an Intelligent Infrastructure is to provide an interoperable platform for bridging the gaps between methodologies, delivery approaches, and continuous business solutions in such enterprises and organizations. This paper discusses what is meant by an intelligent infrastructure, and the state-of-the-art results as delivered by the EXTERNAL project, EU IST-1999-10091. The Intelligent Infrastructure is a platform for distributed, model-generated workplaces, concurrent development and management of models and meta-constructs, methodologies and customer solutions. Focus is on the services provided by the infrastructure, the structures of enterprise knowledge supporting them, and the contributions of Active Knowledge Modelling technology.
\end{abstract}

\section{INTRODUCTION}

Intelligent Infrastructures, having the ability to vary their capabilities and behaviour from experiences and variations in their environments, are becoming very important. An Intelligent Infrastructure is an adaptive platform for developing and delivering architectures and solutions of Extended Enterprises, Virtual Organizations, and Collaborative Business Networks. Its main purpose is to provide continuously evolving operational architectures for achieving solution interoperability and other qualities, such as security, scalability, portability, adaptability, extensibility, and reuse, and to support new approaches for developing and delivering re-computable IT services and solutions.

The Intelligent Infrastructure developed and tested in the EXTERNAL project, IST-1999-10091, introduces many new concepts and innovations, demonstrations and prototypes can be tested from the public portal at: www.external-ist.org. The key contributions of the project are methodologies for the engineering and operation of Extended Enterprises (EE Methodology), and a prototype intelligent infrastructure implementing the methodologies. This intelligent infrastructure is an attempt to realise a test-platform for distributed, model-generated workplaces, concurrent 
development and management of models and meta-constructs, and for developing new methodologies and customer solutions.

The Intelligent Infrastructure is an implementation of some of the Active Knowledge Modelling (AKM) technology core concepts [4]. One core concept is that an active knowledge model can generate an executable task solution, and that executing the task can involve actions to build a detailed knowledge model for generating the next task(s) solution(s). The described implementation is possible because of intrinsic recursive and repeating dependency relationships between metaviews, functional views and other types of enterprise views. Active knowledge models support computerised work, that adapts executable working solutions to individual users, and that can be interacted with and changed in various ways.

The AKM technology performs enterprise integration by inter-relating metamodels and meta-views, and by providing an environment for sharing, cultivating and managing situated or work-supported knowledge, active knowledge that is linked to methods execution. The next chapter describes the concepts, technologies, and services behind the EXTERNAL Intelligent Infrastructure.

\section{FEATURES OF INTELLIGENCE}

Intelligence is normally attributed to the capacities and the capabilities of the human brain, but just as knowledge is externalised and represented in various forms (depending on encoding and media) so is intelligence. An appropriate definition of intelligence of an object or artefact, taken from the Oxford Dictionary, is the "ability to vary its state or actions in response to varying situations and experiences". Applying this to organizations implies a two-way feedback loop between the organization and its infrastructure, and between the infrastructure and its intelligence representation - a closed loop of knowledge management and modifiable actions.

The significant feature of an intelligent enterprise is to which degree the enterprise objectively observes performance and adapts and extends itself to changing operating conditions, and to demands of its stakeholders and customers. Business opportunities and goals drive processes and while knowledge management is driven by innovative change, and should be performed as actions embedded in tasks. Increases in knowledge production and utilization, sustaining a shared meaning in dynamic situations, require an intelligent infrastructure, which is an adaptable generic platform for solutions development, knowledge re-activation and management. An Intelligent Infrastructure is a layered representation of knowledge in various forms and formats, and providing adaptive services to tailor the knowledge to given situations and to the mental models of the humans involved [8].

\section{THE ENTERPRISE ARCHITECTURE}

Enterprise models reflecting operational solution execution will play an important role in the future Networked Organizations. They are a source of precise knowledge for managers, users as well as systems engineers and providers as regards all aspects of user solutions, support and maintenance. 
In figure 1 the infrastructure, the UEMLST box and the two barrels, is the generic part of architecture, gluing the three layers together. The architecture of the infrastructure is, contrary to the remaining architectures, independent of industrial sectors, applications and user solutions. The two barrels are services that are stored in the process repository and can be re-activated and adapted to any given situation.

Holistic view of Enterprise Architect - perspectives of other players $=\boldsymbol{T}$

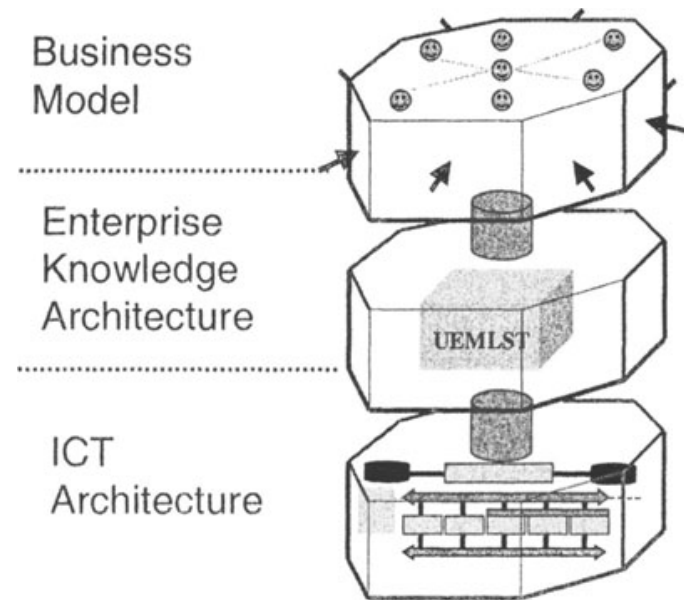

Figure 1
Law, Business rules, principles, Agreed practices and norms

Userviews, Operationalmodels, Meta-models, Language, Structures and Type-hierarchies

Access, capabilities, repositories, services and legacy systems

\subsection{The Business Model Architecture (BMA)}

The concepts at the core of the Business Model Architecture concern the integrated representation of law, business rules, project norms, project and working rules and new rules of collaborative practices. Support to define and assess the adherence to these laws and regulations must be available in an e-Business network. Law, contracted values, rules and obligations are controls on business processes. Business rules are typically agreements on value and risk sharing, division of tasks and responsibilities, and agreements on liabilities and life-cycle support. These mutual expectations and controls must be expressed in a separate business model and correlated with program and project performance indicators. Being able to predict and track performance and the degree of overall and partner success, and assessing the degree of adherence to contract and expected performance goals, are very important properties for successful collaboration. These capabilities all warrant a separate set of models where rules and regulations of working together apart need to be continuously refined and managed, and where the rules of collaboration are continuously changed with the nature of work and local practices and habits. In the EXTERNAL project we did not have objectives nor partners and resources to research the business layer. So on Business Models and Business Architectures we still have a lot of research and experimentation to perform. 


\subsection{The Enterprise Knowledge Architecture (EKA)}

The Enterprise Knowledge Architecture is a set of inter-dependent knowledge dimensions that allows us to separately define, de-couple and manage enterprise knowledge structures and constructs as methodologies and operational solutions. The six major enterprise knowledge dimensions (UEMLST) are composed of:

1. User enterprise views (types and kinds), the kinds of views are comparable to many Extended Enterprises frameworks (Zachman, Cimosa, and GERAM), but the types are not described and explained by these frameworks,

2. Enterprise models and sub-models, and structures of integrated solution models, supporting distributed working,

3. Meta-model definitions of various types and for many kinds of models,

4. Language, core visual constructs form the basis for modelling languages,

5. Structures of meta-model objects and constructs, and finally,

6. Type-hierarchies representing standardized industrial knowledge.

These enterprise knowledge structures are vital for the formation, integration and operation of intelligent enterprises and smart organizations, and must be visually editable and manageable in order to harvest the full benefits of models and visual scenes. The EKA is the most important and common knowledge asset of any enterprise, integrating mental models and augmenting the minds of all employees, for which the EKA is an active visual support system. The EKA represents the nervous centre, the communication central, the enterprise integrator and the representation of all enterprise shared and activated knowledge views! It is the main source of Enterprise Intellectual Capital.

\subsection{The ICT Architecture}

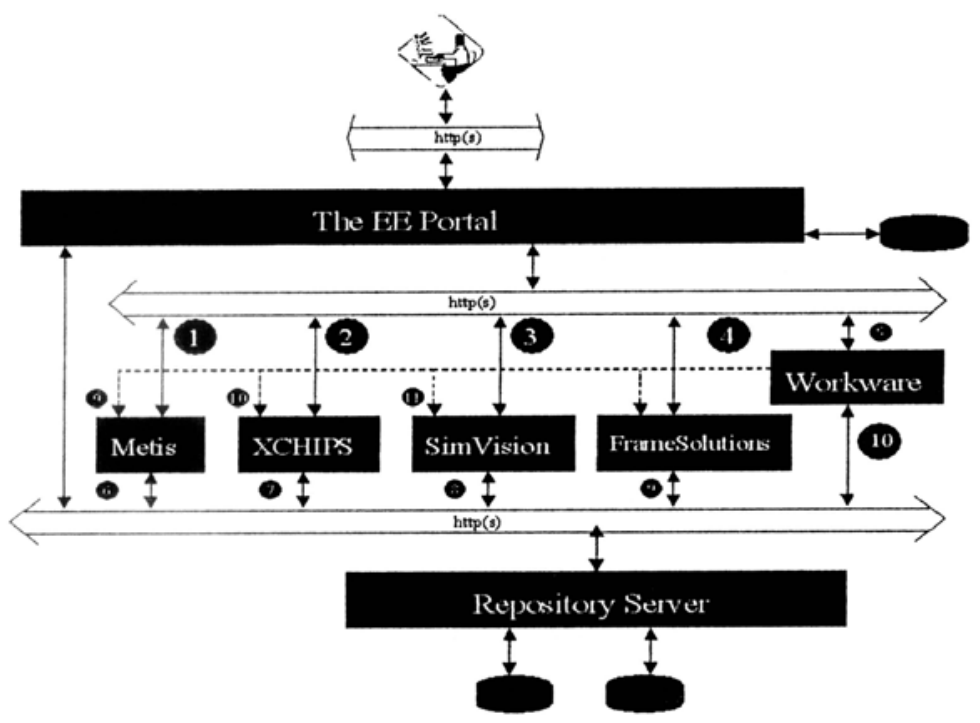

Figure 2 
Figure 2 illustrates the ICT architecture as implemented in the EXTERNAL project. It shows that the user meets the infrastructure through a portal that accesses a set of web services that are made available to the user through the portal. The portal acts as an integrator and as an environment to plug in and perform all kinds of services: software, tasks and applications. The services provided from the portal are services to build knowledge models (Metis), services to cooperate and collaborate (XCHIPS), services to do project simulation (SimVision), services to do work management (Workware) and services to do work execution (FrameSolutions).

\subsubsection{The User Environment Portal Server (UEPS)}

The portal technology used is called the User Environment Portal Server and is developed by Computas. As a front end, UEPS has the main objective of generating a dynamic and personalized user interface, bridging the gaps among the available systems, tools and functions and the users. Combining the Intelligent Infrastructure with techniques enacting dynamic generation of user interfaces, we are able to describe, implement, and deploy software platforms capable of generating operational solutions, driven and managed by knowledge models. The UEPS literally composes the interaction layer using reusable, interoperable software components, called Web Components. Access to the resources is personalized and generated according to policies specified for each project managed by UEPS.

\subsubsection{Client Server Architecture}

The ICT architecture used to implement the portal is based on a multi-tier, distributed client-server architecture. The client tier is the part running on the end user's computer, controlling desktop integration and the user interface. The clients can be thin or thick, from pure HTML/DHTML running in a web browser to a special application such as the METIS modelling clients.

The application-server tier is hosted on a high-performance, transactional /multiuser application server. All knowledge components execute on the application server, which takes care of persistence, transactions and security. The application server tier also includes the web server for use with web clients.

The enterprise and data storage tier consists of the persistent storage system(s) being a file-based repository, an object database and/or a relational database for storage of administrative and operational data.

\section{THE INFRASTRUCTURE SERVICES}

The intelligent infrastructure appears to its users as a portal with a set of services that allows them to develop, deploy and manage model driven solutions.

The most interesting services are shortly described below:

1. Modelling and Meta-modelling services

The Metis modelling environment is used to build active knowledge models used to support work management and work execution, as well as other business aspects of the enterprise or projects. 
Metis supports both modelling and meta-modelling - the meta-modelling capabilities allow users to add new or modify existing language representations. This is important in order to represent knowledge structures as required by the Enterprise Knowledge Architecture.

2. Simulation and Training services

These are services to support different kinds of simulation of Extended Enterprise models. The SimVision tool was used to simulate project and process performance and execution.

3. Work Management services

The work management service is based on Workware and is performed through the concept of shared work-lists. A work-list consists of a set of tasks to be performed with priorities, due dates etc. A task represents a piece of work that needs to be done. The work management service also supports enactment of the tasks. Task planning and work monitoring services are supported through the collaborative environment XCHIPS, allowing multiple users to cooperate on these issues.

4. Work Execution services

The EXTERNAL infrastructure uses FrameSolutions to implement its knowledge model dependent execution services. These are services that require a high degree of operational support to ensure that the work is done according to a given procedure and/or according to certain laws and rules. FrameSolutions is a framework for building rule-driven just-in-time work process execution support.

5. Collaboration services

The collaboration service is a server application that provides support for synchronous and asynchronous work collaboration. Synchronous collaboration will be supported both through the use of NetMeeting or similar third-party products that support many of the tools we will find at customer sites, and the use of the XCHIPS environment that includes a set of components that support synchronous work

6. Resource management service

The EE infrastructure will support a user role model. This makes it possible to group users who have the same needs and responsibilities. The role is given access rights to the desired part of a model rather than the users themselves.

Due to the personal nature of the user environment (the portal) it is however also possible to give individual users access rights to specific structures and services in the portal.

7. Integration services

The intelligent infrastructure has a lot of built-in services, but still there will always be a need to adapt interfaces to other systems or data sources. 
The intelligent infrastructure offers several ways to interface to other systems - the COM approach is one that makes it simple to interface to the Microsoft world. The Metis modelling tool has implemented a service that makes it possible for users to import and visualize relevant data from databases and use those data for further modelling. The service is generic all it requires is a specification of where to find the data and how to map the database content to instances in the importing model.

\section{MODEL DRIVEN OPERATIONAL SOLUTIONS}

The intelligent infrastructure is an environment for developing, deploying and performing model driven operational solutions. In operation the first that meets the eye of the user is the portal driven by the User Environment Portal Server (UEPS). It acts as an integrator that can plug and play services, and its behaviour is key to the perception of the actual operational solutions as intuitive and well-behaved. An EXTERNAL scenario may act as an example of a model driven operational solution.

\subsection{The Project Management scenario}

\section{Modelling.}

The scenario starts with building a project model using the Metis modelling services. The model contains the project work breakdown structure modelled as tasks, supplemented with human resources, information carriers and tools. The model may also include other entities relevant to the project such as requirements, goals, risks, etc. The project model may be rich in details or poor in details, but it is important that it includes allocation of resources for planning and operational purposes.

\section{Simulation.}

In the case of a large project with a lot of detailed activities, it may be valuable to simulate (using SimVision) the predicted time to complete the project, the total effort to do the project etc. and as a result of the analysis, go back to the model and modify it.

\section{Work management.}

Finally, when the model is completed, the work management services (Workware) are initiated (within the portal) for operational support of the project in question, and the actual project may go into operation with work management support. New tasks that appear when the project is in operation are created within the work management service and are reflected in the model upon execution. The initial work management user interface and tasks are generated completely as a result of the model content.

\section{Work execution.}

A task may be enacted by the work management service, either by invoking a link or by starting the just-in-time execution support engine (FrameSolutions). The execution support is represented as a process/execution model defining what to do to execute task actions, and to adopt the rules controlling the execution. The result is a 
workplace integrated in the portal that gives the user the required execution support, and that has the required services to be tailored to personal needs.

\section{Coordination.}

When need for coordination is apparent the work management service allows the users to start the collaborative work management services in XCHIPS to support this. These are services to ease planning and monitoring the tasks. Then one or a few tasks may be opened for discussion and corrections. The corrected tasks are saved to the repository and ready for continued operation.

\section{Other capabilities.}

At any time the project model may be viewed in a model viewer showing the current status of the tasks. At any time the project model may be simulated using the simulation services.

\section{SUMMARY AND CONCLUSIONS}

The Intelligent infrastructure developed as part of the EXTERNAL project is a first step towards an intelligent infrastructure product. This is an infrastructure that has started to use rules and models to control its behaviour, and as such is able to adapt to different operational situations. The infrastructure will be further developed in future EU projects. The next generation of the model driven intelligent infrastructure will be named The AKM Intelligent Infrastructure.

\section{REFERENCES}

Main references are made to the EXTERNAL IST 1999-10091, Deliverable D7 Extended Enterprise Infrastructure. http://www.external-ist.org/.

Dehli, E. 2002, Model-Driven Business Operations, ECSQ 2002, Springer LNCS Series, Springer-Verlag, Berlin Heidelberg New York

Lillehagen, F. \& Krogstie, J. 2002, Active Knowledge Models and Enterprise Knowledge Management, in Enterprise Inter- and intra-organizational integration, Kluwer Academic Publishers, Kosanke K. et al (ed.)

Computas 2003, Metis, http://www.computas.com/metis/

Lillehagen F. 2003, The foundation of the AKM Technology, To be published at CE 2003, Madeira

Lillehagen, F. et al 2002, Utilizing Active Knowledge Models in an Infrastructure for Virtual Enterprises, Infrastructures for Virtual Enterprises, Sesimbra, Portugal, 2002.

Lillehagen, F. \& Krogstie J. 2001. Active Knowledge Models and Enterprise Knowledge Management, ICEIMT workshop, Paris, December 2001

Maestro 2003, Maestro - Model Activiated Enterprise Solutions, Technologies, Research and Operations. Proposal for the EC FP6, delivered April 2003. See also: http://www.maestro-ist.org/

Senge, P. M.: The Fifth Discipline; The Art and Practice of the Learning Organization., Doubleday Currency, New York 1990. 